\title{
MIMO Self-Heterodyne OFDM using SCP with Noise
}

\author{
V. Prasanthi \\ PG Scholar, Vishnu \\ institute of technology \\ vishnupur,bhimavaram
}

\author{
CH. Rama Krishna \\ Assco Professor, Vishnu \\ institute of technology \\ vishnupur,bhimavaram
}

\begin{abstract}
Heterodyning, also known as frequency conversion, used widely in communication engineering to generate new frequencies and move information from one frequency channel to another. In this paper, we are proposing a multipleinput multiple-output (MIMO) self-het OFDM with the adaptive smart carrier position (SCP) technique. At the transmitter, a space-time block code (STBC) is used to produce the coded information symbols to be transmitted on each antenna over the self-het OFDM subcarriers. At the receiver, a simple non-linear detection is adopted at each receiver communication side. The achievable diversity order of such setting is analyzed, and it is found that with the adaptation of SCP technique, the diversity loss in comparison to the conventional coherent MIMO-OFDM can be compensated. Our approach helps in minimising the error floor, phase noise and fading occuring at the reciever.
\end{abstract}

\section{Keywords}

\section{STBC, MIMO, OFDM, SCP}

\section{INTRODUCTION}

In millimetre-wave and terahertz communications, the implementation of stable and low complexity receivers is technically challenging due to instabilities of oscillators and mixers. Hence, significant levels of phase noise are commonly experienced in RF receivers even if advanced frequency stabilization techniques [2], [3] are used.

Conventional orthogonal frequency division multiplexing(OFDM) is a popular technique to combat multipath fading for wireless communications. However, for the millimetre wave and terahertz communications, OFDM is known to have performance degradation due to high levels of residual phase noise at such high frequencies [1]. In 2001, Shoji et. al. proposed self-heterodyne OFDM (self-het OFDM) in [6], [7] as an alternative OFDM technique to compensate these high-level oscillator instabilities in $60 \mathrm{GHz}$ RF bands. In self-het OFDM, the transmitter sends both the local RF carrier and the OFDM subcarriers, and a square-law circuitry (self-mixing) is used at the receiver to down-convert the RF signal. This removes the needs of a local carrier, carrier frequency correction and carrier phase recovery. Since the transmitted local RF carrier phase is synchronous with the OFDM subcarriers, self-het OFDM receivers have theoretically zero phase noise and is extremely stable. However, this advantage comes at the cost of a 50\% reduction in rate. For self-het OFDM, we previously proposed the following two enhancements:

Smart carrier positioning (SCP): It was shown in [11] that the entire OFDM symbol subjects to an outage, if the local RF carrier experiences deep fading. In the SCP, the receiver estimates the optimum RF carrier location (i.e., carrier frequency) using the available channel state information
(CSI), and sends it back to the transmitter. Hence, the SCP can significantly reduce the occurrence of this outage.

Subcarrier pairing scheme [12]: In self-het OFDM, unbalance signal-to-interference noise ratios (SINRs) occur over all subcarriers. The subcarrier pairing is performed across those OFDM subcarriers by grouping good OFDM subcarriers with high SINRs and bad OFDM subcarriers with low SINRs, in order to improve the overall system performance [12].

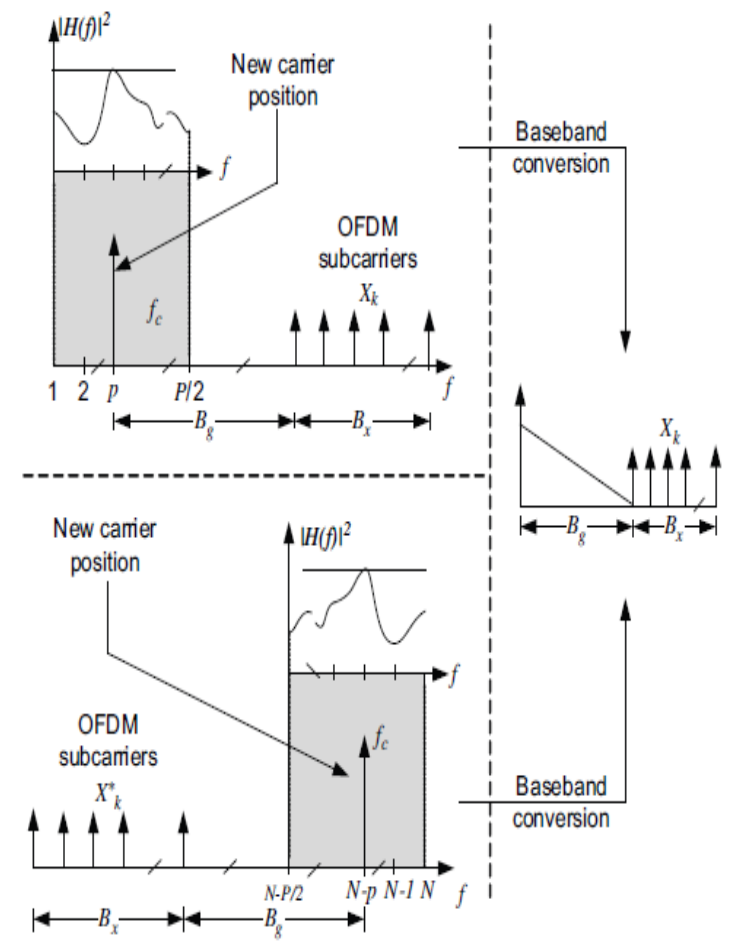

Figure 1: Sub carrier positioning.

In our previous work, we analyzed these enhancements for self-het OFDM over Rayleigh fading channels. However, in millimetre and terahertz channels, the channel fading characteristics may deviate from Rayleigh fading, and more accurate channel models are required for such system performance evaluations. One such model was proposed in [9] by modifying the conventional Saleh-Valenzuela (SV) model [10] and experimentally estimating the relevant parameters for 60 GHzindoor RF channels. In this paper, we adopt this channel modeland we analyze the performance of the enhanced self-het OFDM using SCP and subcarrier pairing over such channels.

OFDM is becoming the chosen modulation technique for wireless communication. With the help of OFDM, sufficient robustness can be achieved to provide large data rates to radio channel impairments. In an OFDM scheme, a large number of orthogonal, overlapping narrow band sub-channels or sub- 
carriers transmitted in parallel by dividing the available transmission bandwidth. Compact spectral utilization with utmost efficiency is achieved with the help of minimally separated sub-carriers. Main attraction of OFDM lies with how the system handles the multipath interference at the receiver end. Multiple inputs and multiple outputs is a method for multiplying the capacity of a radio link using multiple transmit and receive antennas to exploit multipath propagation. MIMO has become an essential element of wireless Communication standards including IEEE802.11n (Wi-Fi), HSPA+ (3G), Wi-MAX (4G), and Long Term Evolution (4G). More recently, MIMO has been applied to power-line communication for 3-wire installations as part of ITU standard and Home Plug AV2 specification. MIMOOFDM is the foundation for most advanced wireless local area network (Wireless LAN) and mobile broadband network standards because it achieves the greatest spectral efficiency and, therefore, delivers the highest capacity and data throughput. Greg Raleigh invented MIMO in 1996 when he showed that different data streams could be transmitted at the same time on the same frequency by taking advantage of the fact that signals transmitted through space bounce off objects (such as the ground) and take multiple paths to the receiver. That is, by using multiple antennas and pre-coding the data, different data streams could be sent over different paths. Raleigh suggested and later proved that the processing required by MIMO at higher speeds would be most manageable using OFDM modulation, because OFDM converts a high-speed data channel into a number of parallel, lower-speed channels.

\section{METHODOLOGY}

Self-heterodyne OFDM (self-het OFDM) is a promising physical layer technique for milli meter-wave and terahertz RF communication due to its simple RF frontend and complete immunity against frequency-offset and phase noise. It shows various techniques as STBC under Altamonte code sequence of Self Heterodyne the performance characteristics based on BER versus SNR. It consists of Altamonte code and Golden code with SCP technique on no phase noise with using alamouti code sequence MIMO self-het OFDM and super heterodynes OFDM. The main drawback of these smart antennas is that they are far more complicated than traditional antennas [MIMO]. This means that fault or problems may be harder to diagnose and more likely to occur. The location of smart antennas [MIMO] needs to be considered for optimal operation. Due to the directional beam that „swings" from a smart antenna locations which are optimal for a traditional antenna is not for a smart antenna [MIMO]. OFDM input signal is given by (1)

$$
s(t)=\Re\left\{\sum_{k=1}^{N_{x}} X_{k} e^{j 2 \pi\left(f_{c}+B_{g}+k \Delta f\right) t}\right\}
$$

OFDM MIMO signal is defined by eq (2)

$$
x(t)=A \cos \left(2 \pi f_{c} t\right)+s(t)_{(2)}
$$

Self-Het OFDM system as an implementation of low complexity stable oscillators is technically difficult. So the performance of self-het OFDM channel undergoes on deep fading. Using Altamonte code sequence on 2X2 MIMO system based self-het OFDM with reduced BER usingwithout diversity loss and side information loss with less amount of fading. Faded signal is given by (3)

$$
r(t)=x(t)+n(t)_{(3)}
$$

Let $h(t)$ be the frequency-selective channel impulse response.

$$
r(t)=h(t) * x(t)+n(t)
$$

In multi-user communication environment, cooperative communication technique enables the neigh bouring mobile users with single antenna to share their antennas in some way for cooperative transmission, which is similar to a distributed virtual multi-antenna transmission environment and combines the advantages of both diversity technology and relay transmission technology. As a result, the spatial diversity gains can be achieved and the system "s transmission performance can be improved in a cooperative In 2X2 MIMO communication system without adding any antennas. 2X2 MIMO self-het OFDM and super heterodyne OFDM using Altamonte code sequence with various bit data values as 16/64/128/256 QAM.. Each pair of transmit-receive antennas provides a signal path from transmitter to receiver. By sending the same information through different paths, multiple independently-faded replicas of the data symbol can be obtained at the receiver end. Hence, more reliable reception is achieved. At the reciever end signal is represented by (5)

$$
y(t)=|r(t)|_{(5)}^{2}
$$

The higher my diversity gain, the lower my Pe. $\mathrm{H}$ is the MIMO channel frequency responses matrix, $\mathrm{Nt}$ is the number of transmit antennas, $\mathrm{Nr}$ is the number of receive antennas, $\mathrm{fc}$ is RF carrier frequency, $\Delta \mathrm{f}$ is OFDM subcarrier spacing, Ns is the number of OFDM subcarriers used to encode information at each transmit antenna, $\mathrm{Ng}$ is the number of subcarriers omitted in each self-het OFDM transmitter, $\mathrm{N}(\mathrm{N}=\mathrm{Ng}+\mathrm{Ns})$ is the size of IFFT/FFT, Bg is the frequency gap between the RF carrier and the first OFDM subcarrier, and Bs is the useful OFDM subcarrier bandwidth. MIMO systems can be defined as: Given an arbitrary wireless communication system, we consider a link in which the transmitting ends as well as the receiving end is equipped with multiple antenna elements as shown in Fig.2. The idea behind MIMO is that the signals on the transmit (TX) antennas at one end and the receive (RX) antennas at the other end are "combined" in such a way that the quality (bit-error rate or BER) or the data rate (bits/sec) of the communication for each MIMO user will be improved. Such an advantage can be used to increase both the network"es quality of service and the operator"s revenues significantly. The parallel-to-serial (PS) conversion, the addition of cyclic prefix (CP), and the digital-to-analogy (DA) conversion are performed to generate the continuous time-domain OFDM symbol.

Space-time block codes (STBC) are orthogonal and can achieve full transmit diversity specified by the number of transmit antennas. The concept of space-time coding has arisen from diversity techniques using smart antennas. By using data coding and signal processing at both sides of transmitter and receiver, space-time coding now is more effective than traditional diversity techniques. The data are constructed as a matrix which has its columns equal to the number of the transmit antennas and its rows equal to the number of the time slots required to transmit the data. At the receiver side, the signals received are first combined and then sent to the maximum likelihood detector where the decision rules are applied. Space-time block codes were designed to achieve the maximum diversity order for the given number of 
transmit and receive antennas subject to the constraint of having a simple linear decoding algorithm. This has made space-time block codes a very popular and most widely used scheme. Altamonte scheme is the basis of the Space Time Coding technique.

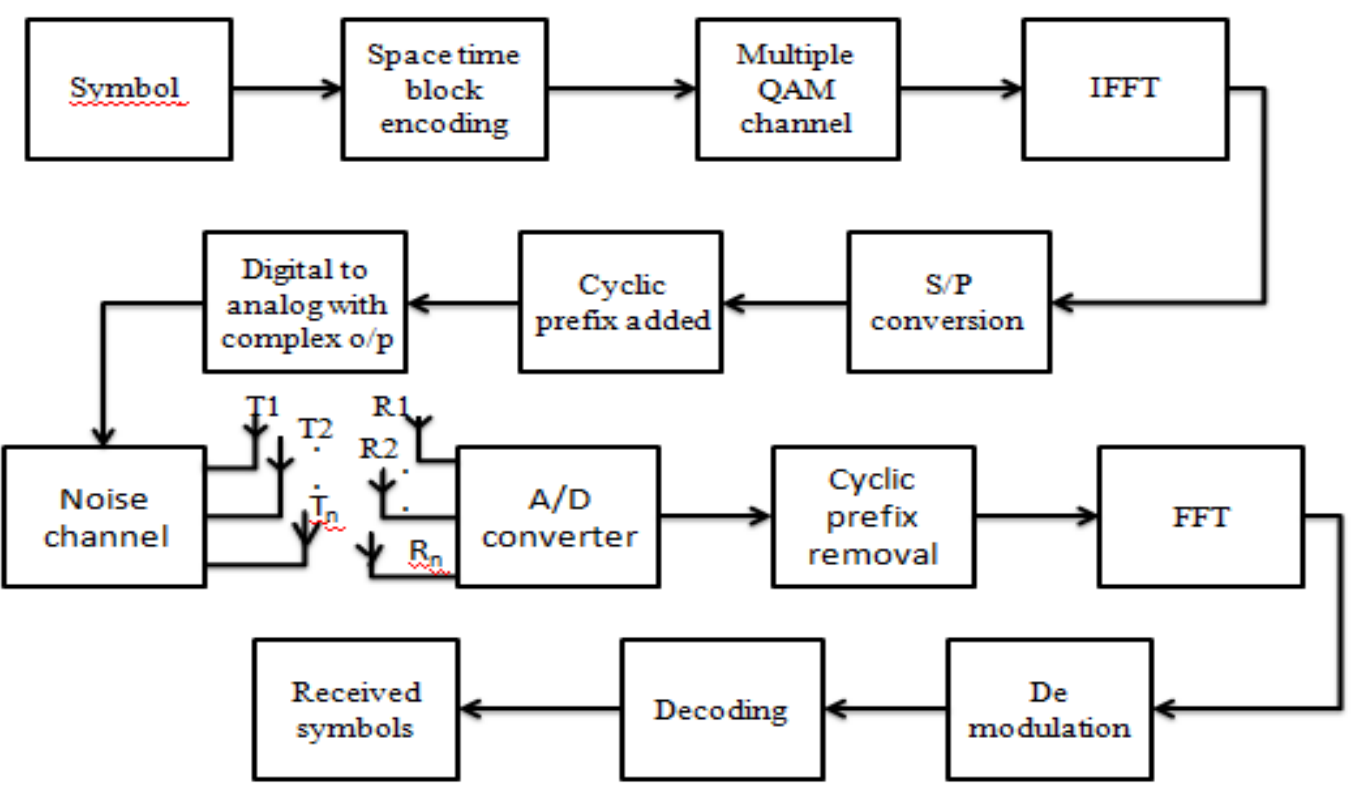

Figure 2: System Model

The mathematical explanation of the scheme with two transmitting and one receiving antennas is also explained here. In this work, a two-branch transmit diversity scheme is implemented. Using two transmit antennas and one receive antenna, the scheme provides the same diversity order as maximal ratio receiver combining (MRRC) with one transmit antenna and two receive antennas. At the transmitter side, a block of two symbols is taken from the source data and sent to the modulator. After that, Altamonte space-time encoder takes the two modulated symbols, in this case called s1 and s2 creates encoding matrix $\mathrm{S}$ where the symbols $\mathrm{s} 1$ and $\mathrm{s} 2$ are mapped to two transmit antennas in two transmit time slots. The encoding matrix is given by the equation.For the encoded matrix QAM approach of modulation is carried with the operations of IFFT and cyclic prefix adding modes. This enhances the phase level of the signal and helps in minimising the phase error. Before transmission of the OFDM signal noise generator was defined and resluted in (4) for proving there will be no fading effect arises in the transmission section.

In the reciever section of OFDM the encoded modulated bits will be reconfigured by the inverse approaches of Cyclic prefix, modulation, FFT and inverse spatial decoding. Where error floor araised in the super heterodyne system will be minimised in the case of self heterodyne system.

\section{RESULTS}

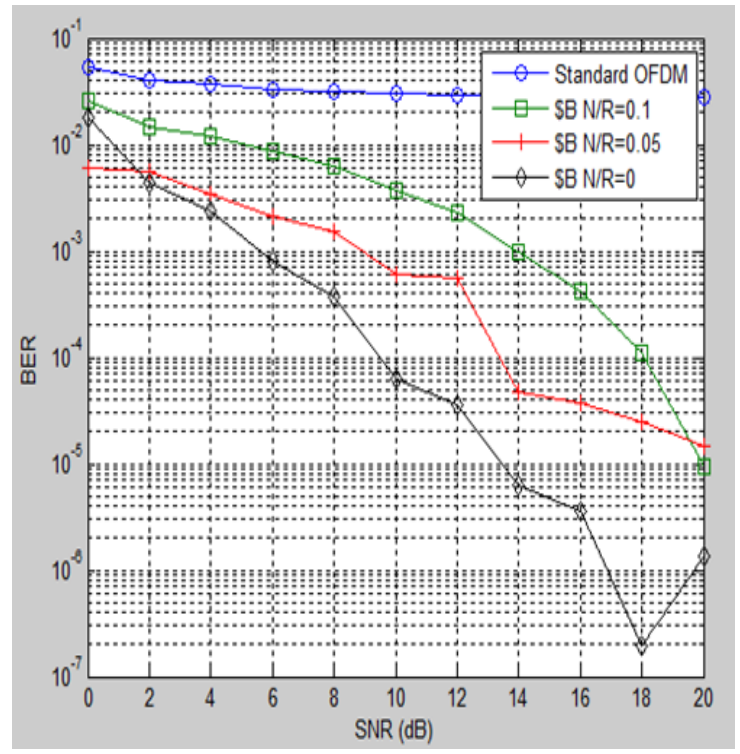

Figure BER performance of SC OFDM MIMO 


\begin{tabular}{|c|c|c|c|c|c|c|}
\hline \multirow{2}{*}{ SNR } & \multicolumn{5}{|l|}{$\begin{array}{l}\text { Alamouti coded MIMO-OFDM with super- } \\
\text { heterodyne in the presence of phase noise }\end{array}$} & \multicolumn{2}{l|}{$\begin{array}{l}\text { Self-Heterodyne with SCP in the presence of } \\
\text { phase noise }\end{array}$} \\
\cline { 2 - 7 } & $\mathrm{NB} / \mathrm{R}=0$ & $\mathrm{NB} / \mathrm{R}=0.05$ & $\mathrm{NB} / \mathrm{R}=0.1$ & $\mathrm{NB} / \mathrm{R}=0$ & $\mathrm{NB} / \mathrm{R}=0.05$ & $\mathrm{NB} / \mathrm{R}=0.1$ \\
\hline 2 & $10^{\wedge}-1.9$ & $10^{\wedge}-1.7$ & $10^{\wedge}-0.7$ & $10^{\wedge}-2.5$ & $10^{\wedge}-2.4$ & $10^{\wedge}-1.9$ \\
\hline 6 & $10^{\wedge}-2.8$ & $10^{\wedge}-2.0$ & $10^{\wedge}-0.8$ & $10^{\wedge}-3.2$ & $10^{\wedge}-2.9$ & $10^{\wedge}-2.2$ \\
\hline 10 & $10^{\wedge}-4.3$ & $10^{\wedge}-2.6$ & $10^{\wedge}-0.9$ & $10^{\wedge}-4.4$ & $10^{\wedge}-3.5$ & $10^{\wedge}-2.6$ \\
\hline 14 & $10^{\wedge}-5.1$ & $10^{\wedge}-2.9$ & $10^{\wedge}-1$ & $10^{\wedge}-5.4$ & $10^{\wedge}-4.5$ & $10^{\wedge}-3.0$ \\
\hline
\end{tabular}

\section{CONCLUSION}

The 2X2 MIMO Self-Het OFDM system provided with cyclic prefix without diversity loss and side information loss with less interference on $2.1 \mathrm{db}$ BER reduced, when it comes to diversity loss in ofdm system the ber is $2.6 \mathrm{db}$ advanced. The performance of the system was compared for the values obtained for both systems, found that the BER Vs SNR for 16QAM is lowest signifying with high performance when there is no phase noise by Self-Het and similarly for phase noise.

It is shown analytically and by simulation that the proposed enhanced non-coherent OFDM techniques offer better BER performance and simpler receiver structures than that of conventional OFDMs. To improve the spectral efficiency, the nonlinear pre-distortion technique is explored for noncoherent OFDMs. Simulation results show that the predistortion aided system can improve the spectral efficiency up to $30 \%$ with a negligible increase of error performance.

\section{REFERENCES}

[1]Chang-Soon Choi, Yozo Shoji, and Hiroyo Ogawa (2007), "Analysis of receiver space diversity gain for millimetre wave self-heterodyne transmission techniques under twopath Channel environments", IEEE, National Institute of Information and Communications Technology (NICT), Japan, pp. 239-0847.

[2]Chang-Soon Choi, Yozo Shoji, and Hiroyo Ogawa (2011), "Implementation of an OFDM Baseband with Adaptive Modulations to Grouped Subcarriers for Millimetre wave wireless Indoor Networks", IEEE Transactions on Consumer Electronics, Vol. 57, No. 4, pp.0098 3063.

[3]Haibing Yang, Peter F.M. Smulders and Matti H.A.J. Herben (2006), "Frequency Selectivity of $60-\mathrm{GHz}$ LOS and NLOS Indoor Radio Channels", IEEE Transactions on Radio Communications, pp. 0-7803-9392-9.

[4]Ho-Jin Song, TadaoNagastuma (2011), "Present and Future of Terahertz Communications", IEEE Transactions on Terahertz Science and Technology, Vol. 1, No. 1, pp. 2156-342.

[5]Nirmal Fernando, Yi Hong, EmanueleViterbo (2013), "Analysis of Self-het OFDM Enhancements for $60 \mathrm{GHz}$ Indoor RF Channels", IEEE, Austrlian Communications Theory Workshop (AusCTW), pp. 4675-7.

[6]Nirmal Fernando, Yi Hong, EmanueleViterbo (2013), "Self-Heterodyne OFDM Transmission for Frequency Selective Channels", IEEE Transactions on Communications Vol. 61, No.5, pp. 0090-6778.

[7]Nirmal Fernando, Yi Hong, EmanueleViterbo (2013), "Subcarrier Pairing for Self-het OFDM", IEEE ICC Transactions on Communications Theory, pp.3122-7.

[8]J.N. Schulman, V. Kolinko, M. Morgan, C. Martin, J. Lovberg, S. Thomas, J. Zinck, and Y.K. Boegeman (2004), "W-Band Direct Detection Circuit Performance with Sb-Heterostructure Diodes", IEEE Microwave and Wireless Components, Vol.14, No.7, pp. 1531-1309.

[9]Yozo Shoji, Chang-Soon Choi, and Hiroyo Ogawa (2007), "Millimeter wave OFDM WPAN system applying adaptive modulation for grouped sub-carriers", IEEE National Institute of Information and Communications Technology (NICT), pp. 239-0847.

[10]Yozo Shoji, Kiyoshi Hamaguchi, and Hiroyo Ogawa (2002), "Millimeter wave Remote Self-Het System for Extremely Stable and Low Cost Broad Band Signal Transmission", IEEE Transactions on Microwave Theory and Techniques, Vol.50, No.6, pp. 0018-9480. 